\title{
Acyl homoserine lactone-based quorum sensing in a methanogenic archaeon
}

\author{
Guishan Zhang ${ }^{1}$, Fan Zhang ${ }^{1}$, Gang Ding ${ }^{2}$, Jie Li $^{1}$, Xiaopeng Guo ${ }^{1}$, Jinxing Zhu ${ }^{1}$, \\ Liguang Zhou ${ }^{1}$, Shichun Cai ${ }^{1}$, Xiaoli Liu ${ }^{1}$, Yuanming Luo ${ }^{1}$, Guifeng Zhang ${ }^{3}$, \\ Wenyuan $\mathrm{Shi}^{4}$ and Xiuzhu Dong ${ }^{1}$ \\ ${ }^{1}$ State Key Laboratory of Microbial Resources, Institute of Microbiology, Chinese Academy of Sciences, \\ Beijing, PR China; ${ }^{2}$ Institute of Medicinal Plant Development, Chinese Academy of Medical Sciences and \\ Peking Union Medical College, Beijing, PR China; ${ }^{3}$ State Key Laboratory of Biochemical Engineering, Institute \\ of Process Engineering, Chinese Academy of Sciences, Beijing, PR China and ${ }^{4}$ Department of Microbiology, \\ Immunology and Molecular Genetics, University of California, Los Angeles, CA, USA
}

\begin{abstract}
Acyl homoserine lactone (AHL)-based quorum sensing commonly refers to cell density-dependent regulatory mechanisms found in bacteria. However, beyond bacteria, this cell-to-cell communication mechanism is poorly understood. Here we show that a methanogenic archaeon, Methanosaeta harundinacea $6 \mathrm{Ac}$, encodes an active quorum sensing system that is used to regulate cell assembly and carbon metabolic flux. The methanogen 6Ac showed a cell density-dependent physiology transition, which was related to the $A H L$ present in the spent culture and the fill gene-encoded $A H L$ synthase. Through extensive chemical analyses, a new class of carboxylated AHLs synthesized by Fill protein was identified. These carboxylated AHLs facilitated the transition from a short cell to filamentous growth, with an altered carbon metabolic flux that favoured the conversion of acetate to methane and a reduced yield in cellular biomass. The transcriptomes of the filaments and the short cell forms differed with gene expression profiles consistent with the physiology. In the filaments, genes encoding the initial enzymes in the methanogenesis pathway were upregulated, whereas those for cellular carbon assimilation were downregulated. A luxI-luxR ortholog fill-filR was present in the genome of strain 6Ac. The carboxylated AHLs were also detected in other methanogen cultures and putative fill orthologs were identified in other methanogenic genomes as well. This discovery of AHL-based quorum sensing systems in methanogenic archaea implies that quorum sensing mechanisms are universal among prokaryotes.
\end{abstract}

The ISME Journal (2012) 6, 1336-1344; doi:10.1038/ismej.2011.203; published online 12 January 2012 Subject Category: microbe-microbe and microbe-host interactions Keywords: carboxylated acyl homoserine lactones; fill-encoded AHL synthase; methanogenic archaea; physiology transition; quorum sensing

\section{Introduction}

Quorum sensing is a well-characterized gene-regulatory mechanism that coordinates diverse social behaviours in bacteria, such as biofilm formation, antibiotic resistance, production of luminescence, exotoxins and exopolysaccharides, and uptake of extracellular DNA (Hastings and Greenberg, 1999; Waters and Bassler, 2005). In this process, bacteria communicate through secreted signal molecules or autoinducers, which are involved in gene regulation. The Gram-negative bacteria use acyl homoserine lactones (AHLs) as a quorum sensing signal to achieve regulation through the luxI-luxR homolog

Correspondence: X Dong, State Key Laboratory of Microbial Resources, Institute of Microbiology, Chinese Academy of Sciences, No.1 West Beichen Road, Beijing 100101, PR China.

E-mail: dongxz@im.ac.cn

Received 26 April 2011; revised 21 November 2011; accepted 28 November 2011; published online 12 January 2012 system, whereas Gram-positive bacteria use small peptides as autoinducers. Despite the ubiquity of quorum sensing systems in bacteria, quorum sensing has yet to be conclusively identified in archaea, except that a bacterial biosensor was reported to detect potential quorum sensing signals from a halophilic archaeon (Paggi et al., 2003) and genome analyses suggest that LuxS-based AI-2 signalling may be present (Sun et al., 2004).

We previously isolated an obligate aceticlastic methanogenic archaeon, Methanosaeta harundinacea 6Ac, from the up-flow anaerobic sludge bed granules in an anaerobic digester (Ma et al., 2006). Intriguingly, this methanogen shows a cell densitydependent cell assembly (as described below), making it a good model system for the study of quorum sensing behaviour in archaea. In the present study, a bacterial luxI-luxR-like circuit was observed in M. harundinacea 6Ac and a luxI homolog called the fill gene was shown to encode AHL 
synthase. This archaeal enzyme synthesized a group of carboxylated AHL with 10-14 carbon atoms in the acyl chain. Furthermore, these carboxyl-AHLs acted as the quorum sensing signal in regulating the physiology of $M$. harundinacea 6Ac. Therefore this methanogenic archaeon uses a quorum sensing system similar to that used by Gram-negative bacteria.

\section{Materials and methods}

Methanogen strain and growth conditions

M. harundinacea 6Ac was maintained in our laboratory and routinely cultured in a pre-reduced basal medium containing $50 \mathrm{mM}$ sodium acetate, yeast extract $(0.05 \%, \mathrm{w} / \mathrm{v})$ and peptone $(0.05 \%, \mathrm{w} / \mathrm{v})$ as described previously (Doddema and Vogels, 1978; Ma et al., 2006). To promote the formation of filaments, the acetate in growing cultures was replenished up to three times with $50 \mathrm{mM}$ sodium acetate.

\section{$\mathrm{N}$-acyl-homoserine lactone bioassay}

Agrobacterium tumefaciens NTL4, which carries the plasmid pZLR4, was used as an $N$-acyl-homoserine lactone reporter. Plasmid pZLR4 contains a traG::lacZ fusion and traR (Steindler and Venturi, 2007). The plate assay for detection of AHLs was performed as described previously (Hwang et al., 1994). Briefly, AB agar containing 0.2-0.5\% glucose and $40 \mu \mathrm{g} \mathrm{ml}^{-1} \mathrm{X}$-gal was poured into Petri dishes, then half of the plate was overlaid with $0.7 \%$ agar containing the reporter strain NTL4, whereas another half was overlaid with only $0.7 \%$ agar as a blank. After the overlay solidified, $5 \mu \mathrm{l}$ of the test liquid was spotted onto to the soft agar on both halves of the plate. The plates were incubated at $28{ }^{\circ} \mathrm{C}$ for $12-48 \mathrm{~h}$. Formation of blue spots indicated the presence of AHLs in the test sample. AHLs in the spent culture were quantified according to the method of Cha et al. (1998).

\section{Cloning of fill gene, and expression and purification} of Fill protein

The genomic DNA of $M$. harundinacea 6Ac was extracted as described previously (Zhou et al., 1996) and was used as template for PCR amplification of ORF00438 (GenBank no. HQ188282) (fill). The primer pair shown in Supplementary Table S3 was used for PCR amplification of the fill gene. PCR amplification was performed using Pfu DNA polymerase (Promega, Madison, WI, USA) for 30 cycles, with each cycle consisting of denaturation at $95{ }^{\circ} \mathrm{C}$ for $45 \mathrm{~s}$, annealing at $59{ }^{\circ} \mathrm{C}$ for $1 \mathrm{~min}$ and elongation at $72{ }^{\circ} \mathrm{C}$ for $5 \mathrm{~min}$. The PCR product was purified using the $3 \mathrm{~S}$ Spin Agarose Gel DNA Purification kit (Shanghai Biocolor Bioscience and Technology Company, Shanghai, China) and cloned into the pET28a vector for expression in the Escherichia coli
Rosseta (DE3) strain. The FilI protein was purified by HisTrap HP and Q ion exchange column chromatography (GE Healthcare, Piscataway, NJ, USA).

\section{Enzymatic synthesis of AHLs by FilI}

The principle of in vitro enzymatic synthesis of AHLs was described elsewhere (More et al., 1996; Val and Cronan, 1998). Briefly, S-adenylmethionine was the substrate for the homoserine lactone moiety and the cell-free extract of strain 6Ac was the source of acyl-carrier proteins (ACPs), according to the presence of a $\beta$-ketoacyl-acyl carrier protein synthase gene fabH (Mhar_2367) in the genome, although no ACP homologue is found in the genome. Cells in mid-log phase were collected by centrifugation, resuspended in TEDG buffer $(10 \mathrm{mM}$ Tris-HCl (pH 8.0), 0.1 mM EDTA, $0.1 \mathrm{~mm}$ dithiothreitol, 5\% glycerol), and disrupted by ultrasonication for $30 \mathrm{~min}$ on ice. The cell lysate was centrifuged at $20000 \mathrm{~g}$ for $1 \mathrm{~h}$ and the supernatant of about $5 \mathrm{mg}$ protein per $\mathrm{ml}$ was used for ACPs. Fill synthesis reactions $(200 \mu \mathrm{l})$ were performed in microfuge tubes at $37^{\circ} \mathrm{C}$ for $30 \mathrm{~min}$. The reaction mixture contained $10 \mathrm{~mm}$ Tris-HCl (pH 7.4), $330 \mathrm{~mm}$ NaCl, $15 \%$ glycerol, $0.7 \mathrm{mM}$ dithiothreitol, $2 \mathrm{mM}$ EDTA, $25 \mathrm{mM}$ $\mathrm{MgSO}_{4}, \quad 0.1 \mathrm{mM} \quad \mathrm{FeSO}_{4}$, and $0.15 \mathrm{mM} S$-adenylmethionine and $60 \mu \mathrm{l}$ cell-free extract of strain 6Ac, unless otherwise indicated. The reaction was initiated by adding $6 \mu \mathrm{g}$ per ml of $\mathrm{His}_{6}$-FilI and stopped by adding three volumes of ethyl acetate. The ethyl acetate phase was collected and dried in a rotary vacuum evaporator at $30^{\circ} \mathrm{C}$. The residue was re-suspended in methanol for purification of AHLs.

Purification of Fill-synthesized AHLs

AHLs synthesized by FilI were purified using an Agilent ZORBAX Extend-C18 Column $(4.6 \times 250 \mathrm{~mm}, 5 \mu \mathrm{m}$ particle size $)$ on Agilent's 1100 Series HPLC, and monitored with a Finnigan SpectraSYSTEM UV6000LP PDA Detector and LCQ Deca XPplus ion-trap mass spectrometer (ThermoFinnigan, San Jose, CA, USA) (Ortori et al., 2007). The purification parameters were as follows: column temperature $15^{\circ} \mathrm{C}$; mobile phase-A, water with $0.01 \%$ trifluoroacetic acid; mobile phase-B, acetonitrile with $0.01 \%$ frifluoroacetic acid; flow rate, $0.8 \mathrm{ml} \mathrm{min}^{-1}$; gradient profile, linear increase of phase-B from 30 to $90 \%$ over 5-65 min. The column was re-equilibrated for a total of $15 \mathrm{~min}$ prior to $50 \mu \mathrm{l}$ loading. The three AHL compounds were collected in the elute fractions with a retention time of 25-35 min.

Mass spectral determination of AHLs

Electrospray ionization mass spectra were recorded in the positive ionization mode using a LCQ Deca $\mathrm{XP}^{\text {plus }}$ ion-trap mass spectrometer (Thermo-Finnigan) 
(Cataldi et al., 2009). Samples in 50\% methanol were infused directly into the source at $5 \mu \mathrm{lmin}{ }^{-1}$ using a syringe pump. The transfer capillary temperature and spray voltage were set at $275^{\circ} \mathrm{C}$ and $5.5 \mathrm{kV}$, respectively (Sharif et al., 2008). The sheath gas flow rate was set to 12 arbitrary units and the tube lens offset was set to $25 \mathrm{~V}$. For $\mathrm{MS}^{\mathrm{n}}$ analysis, selected precursor ions were isolated with a width of $3 \mathrm{~m} / \mathrm{z}$, and the collision energy was optimized to obtain the stable and entire product ion spectra. Based on above mass spectrometric conditions, the specific fragments of the AHL standards ( $N$-capry-

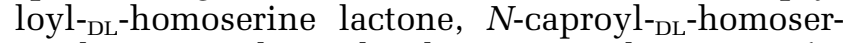
ine lactone, $N$-lauroyl- ${ }_{\mathrm{DL}}$-homoserine lactone, $N$ - $(\beta$ ketooctanoyl)-L-homoserine, $N$-heptanoyl- ${ }_{\text {DL }}$-homoserine lactone and $N$-( $\beta$-ketocaproyl)- ${ }_{\mathrm{DL}}$-homoserine lactone, $\mathrm{N}$-tetradecanoyl-${ }_{\mathrm{DL}}$-homoserine lactone and $\mathrm{N}$-decanoyl- ${ }_{\mathrm{DL}}$-homoserine lactone; Sigma-Aldrich, St Louis, MO, USA) were further characterized. The core homoserine lactone (HSL) moiety was monitored at $\mathrm{m} / \mathrm{z} 102.05$ and electrospray ionization mass spectrometry (ESI-MS)/MS spectra were optimized over the range $\mathrm{m} / \mathrm{z}$ 50-110. Using ESI (positive ion mode) coupled with Fourier transform ion cyclotron resonance MS APEX IV (ESI-FT-ICR; Bruker Daltonics, Billerica, MA, USA), high-resolution data of the compounds in methanol were determined. The Bruker Compass Data Analysis software (version 4.0) was used for data acquisition and processing (Cataldi et al., 2008).

\section{NMR spectroscopic analysis}

NMR spectra were acquired with a Varian Mercury $600 \mathrm{MHz}$ NMR spectrometer (Varian Corp., Palo Alto, CA, USA) operating at $600.13 \mathrm{MHz}$ proton frequency by using a $3 \mathrm{~mm}$ inverse geometry broadband probe head equipped with an actively shielded z-gradient coil $\left(907\left({ }^{1} \mathrm{H}\right)\right.$ 7.3/9.8 $\mathrm{ms} \mathrm{CDCl}_{3} / 0.01 \mathrm{M}$ sodium carbonate) (Pearson et al., 1995; Frommberger et al., 2005). ${ }^{1} \mathrm{H}$ NMR spectra (AQ, $5.23 \mathrm{~s}$; relaxation delay, $0.1 \mathrm{~s}$; exponential line-broadening, $0.3 \mathrm{~Hz}$ ) were recorded with 20-907 pulses.

\section{Cell protein concentration determination}

Protein concentrations were determined using the BCA Protein Assay kit (Thermo Scientific, Rockford, IL, USA), using bovine serum albumin as the calibration standard.

qPCR experiments

Quantitative PCRs (qPCRs) were performed in eight strip PCR tubes (Axygen, Union City, CA, USA), and the reaction signals were generated by the binding of SYBR green to double-stranded DNA. All qPCR experiments were performed using an ABI Prism 7000 Sequence Detection System (Applied Biosystems, Foster City, CA, USA). The primers used for quantitative real-time PCR analysis are listed in Supplementary Table S3 and were designed using the Premier express 2.0 software, Primer Premier 5.0 and Oligo 6.0. Two pairs of primers, 16SRTF/16SRTR and filiRTF/ filiRTR, amplified the 16S rRNA and the ORF00438 gene of strain 6Ac, respectively. The specificity of the primer sets was confirmed by sequencing the amplicons (215 bp). Plasmids bearing the 16S rRNA (AY970347) or the fill gene of strain 6Ac were used as standards. Plasmids were extracted by the Tianprep Mini Plasmid Kit (Tiangen Biotech, Beijing, China) and purified using the 3S Spin Agarose Gel DNA Purification kit (Shanghai Biocolor Bioscience and Technology Company). The DNA preparations were quantified by using a NanoDrop ND-1000 UV-Vis Spectrophotometer (Thermo Scientific, Wilmington, DE, USA). The 16S rRNA copy number was calculated as described previously (Yu et al., 2005).

qPCR experiments were performed using the 16SRTF/16SRTR and filiRTF/filiRTR primer sets (Supplementary Table S3). Each qPCR mixture contained $12.5 \mu \mathrm{l}$ of $2 \times$ SYBR green mastermix (Applied Biosystems), $1 \mu \mathrm{l}$ of DNA template, $100 \mathrm{nM}$ of each primer and $\mathrm{ddH}_{2} \mathrm{O}$ to a final volume of $25 \mu \mathrm{l}$. The PCR was initiated at $50{ }^{\circ} \mathrm{C}$ for $2 \mathrm{~min}$ to optimize AmpErase uracil- $N$-glycosylase activity, followed by denaturation at $95{ }^{\circ} \mathrm{C}$ for $10 \mathrm{~min}$ and 40 cycles of amplification as follows: DNA denaturation at $95^{\circ} \mathrm{C}$ for $30 \mathrm{~s}$, primer annealing at $57^{\circ} \mathrm{C}$ for $40 \mathrm{~s}$ and elongation at $72{ }^{\circ} \mathrm{C}$ for $40 \mathrm{~s}$. Fluorescence data were collected during the elongation steps. The reactions were all performed in three replicates.

\section{Microarray procedure}

For transcriptome studies, $M$. harundinacea 6Ac was grown in $30 \mathrm{~mm}$ sodium acetate to prevent conversion of short cells into filaments, and growth of cultures was monitored by determining methane production. Purified $\mathrm{N}$-carboxyl- $\mathrm{C}_{12}$-HSL $(\mathrm{m} / \mathrm{z} 346)$ (final concentration, $50 \mathrm{nM}$ ) was added to triplicate cultures during the mid-log growth phase. After further incubating until the late-logarithmic phase, the formation of filaments was verified before extracting total RNAs with the TRIZol reagent (Invitrogen, Carlsbad, CA, USA) and further purification using the RNeasy Mini kit (Qiagen, Hilden, Germany). The Agilent Low Input Quick Amp Labeling kit (Agilent Technologies, Santa Clara, CA, USA) was used to synthesize cDNA from the total RNA samples and subsequently produce the amino allyl-modified cDNA according to the manufacturer's instructions. The amino allyllabelled cDNA was then linked to Cy3 NHS ester (GE Healthcare). The fluorescently labelled cDNA was hybridized with an Agilent M. harundinacea 6Ac Custom $8 \times 15 \mathrm{~K}$ Microarray according to the manufacturer's protocol. The Feature Extraction Software (Agilent Technologies) was used for data acquisition and GeneSpring (Agilent Technologies) was used for further data processing according to published procedures (Gobert et al., 2009). To validate the microarray data, qPCRs were performed using the primer pairs listed in Supplementary Table S3. 
The PCR mixtures included $200 \mathrm{nM}$ primers, 1$100 \mathrm{ng}$ of cDNA and $10 \mu \mathrm{l}$ of $2 \times$ SYBR Green PreMix (TaKaRa, Dalian, China). The parameters were $95{ }^{\circ} \mathrm{C}$ for $30 \mathrm{~s}$, followed by 40 cycles of $95^{\circ} \mathrm{C}$ for $5 \mathrm{~s}, 60{ }^{\circ} \mathrm{C}$ for $15 \mathrm{~s}$ and $72{ }^{\circ} \mathrm{C}$ for $31 \mathrm{~s}$.

\section{Results}

Cell density-dependent morphology change of

M. harundinacea

During growth in the up-flow anaerobic sludge bed granules, $M$. harundinacea 6 Ac formed rigid, manelike filaments encasing multiple cells in a common shell. However, upon sub-culturing these cells in $50 \mathrm{mM}$ acetate, the optimal concentration for growth, the cell assemblages became substantially shorter with less than three linked cells. As cell density increased upon supplementing the spent cultures with additional substrate, rigid mane-like filaments (Figures 1a and b) re-formed. Interestingly, the shorter ones (inserts in Figures 1a and b) could become filaments if they were concentrated by centrifugation, or resuspended in the conditioned media from the spent filament cultures. Furthermore, the filament cells emitted intensive fluorescence when excited at $420 \mathrm{~nm}$, owing to oxidation of the methanogen-specific cofactor $\mathrm{F}_{420}$, whereas this ability was abrogated in the short cell morphology (Figure 1a). Similarly, colony formation was promoted on agar plates containing conditioned medium (Figure 1c), whereas colonies were rarely observed on plates containing only the basic medium. These observations suggest that M. harundinacea used extracellular signals to control cell assembly in a cell density-dependent manner, which resembles quorum sensing behaviours reported in bacteria.
Identification of the possible quorum sensing signals in M. harundinacea

To determine the identity of the extracellular signal produced by $M$. harundinacea, medium from cell-free spent cultures of filamentous cells was extracted with ethyl acetate and the ethyl acetate extract was tested for various bioactive molecules. Interestingly, the 300-1500 times concentrated solutions derived from the ethyl acetate extracted spent media triggered the expression of an AHL reporter strain, Agrobacterium sp. NTL4, suggesting the possible presence of AHL-like molecules. By using $\beta$-ketooctanoyl-L-homoserine lactone as the standard, AHL(s) at concentrations of 100-500 nM and 10-27 $\mu \mathrm{M}$ were determined in the short cell and filament cultures, respectively. Next, the ethyl acetate extract was examined by tandem ESI-MS and compounds with a molecular ion $\mathrm{m} / \mathrm{z}$ of 318.2 , 274.2, 284.2, 346.2, 374.2 and 302.3 were observed in a relatively high abundance (Supplementary Figure S1a). These compounds all produced a diagnostic HSL ion $m / z \quad 102$ in their tandem ESI-MS (Supplementary Figure S1a), similar to the chemical $\beta$-ketooctanoyl-L-homoserine lactone (Supplementary Figure S1b). This result confirmed the presence of AHLs in the sample. However, the AHL level in the medium was too low to be purified for further structural identification.

\section{Determination of an AHL synthase gene, filI, in}

M. harundinacea

By analogy with the bacterial systems, LuxI would be the key enzyme for this process. Thus, we used the well-defined bacterial luxI orthologues (Supplementary Figure S2) as probes to query the completed genome sequence of $M$. harundinacea using TBLASTN. The best match was ORF00438
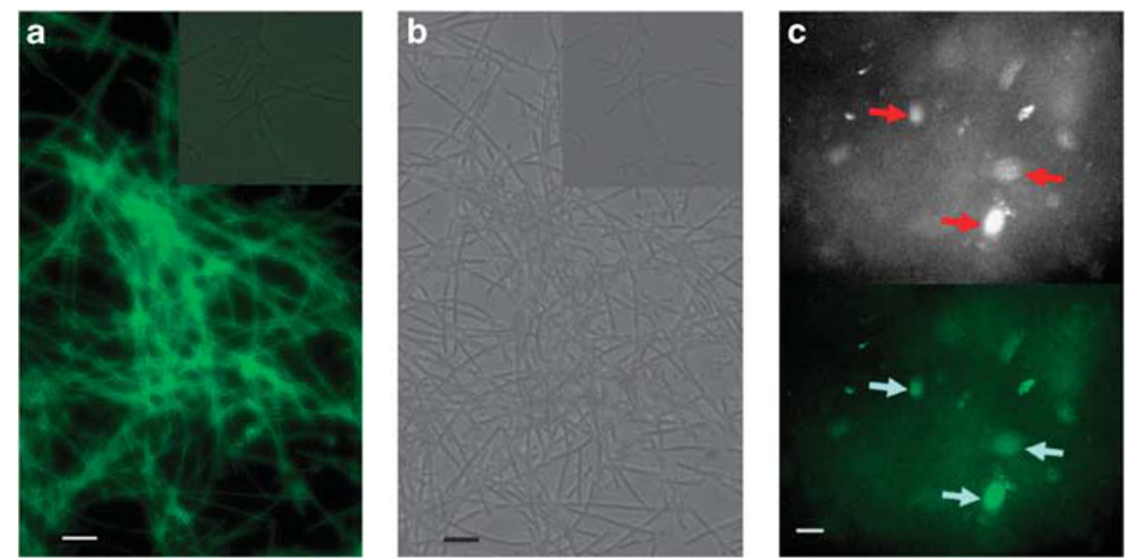

Figure 1 Induction of filaments and colony formation by the filamentous spent culture of $M$. harundinacea. (a) A microscope image of the filaments in conditioned medium with spent culture of filamentous cells showing fluorescence at $420 \mathrm{~nm}$, whereas the short cells (insert) show no fluorescence. Bar, $5 \mu \mathrm{m}$. (b) Phase-contrast microscopic image of the filaments in conditioned medium with spent culture of filamentous cells and short cells (insert). Bar, $5 \mu \mathrm{m}$. (c) Microscope images of colonies forming on conditioned agar medium with spent culture of filamentous cells. The arrows indicate the presence of colonies using a light microscope (upper) and illumination at $420 \mathrm{~nm}$ (lower). Bar, $1 \mathrm{~mm}$. 

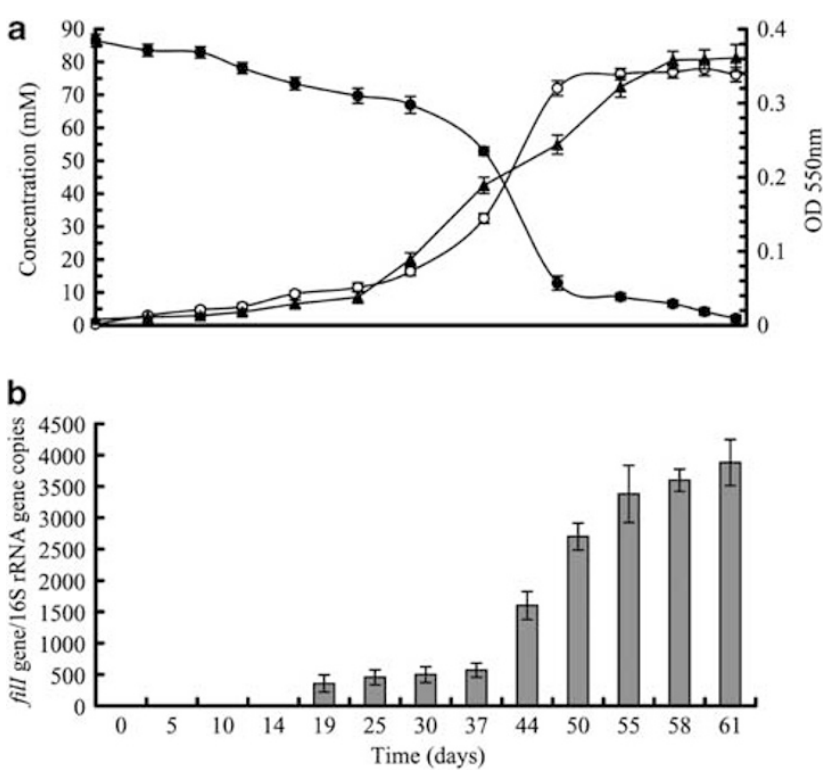

Figure 2 fill expression during growth. (a) Culture absorption (open circles) and methane formation (triangles) by M. harundinacea 6Ac during growth on acetate (closed circles). (b) Copies of fill mRNA per 16S rRNA gene estimated by qPCR using a pair of fill-specific primers listed in Supplementary Table S3. Copy numbers were calculated from duplicate cultures. The error bars show standard deviation.

(HQ188282), which is annotated as a 'multi-sensor signal transduction histidine kinase', and the CHASE 4 domain of the protein shows 39.6\% identity to AhlI, the LuxI autoinducer synthase of Erwinia chrysanthemi (GenBank accession number AAM46699). Based on this result, ORF00438 was renamed as fill to reflect its role in inducing the filamentous morphology. The transcript level of filI was examined during growth and it increased with cell density, which is typical of many bacterial quorum sensing-regulated genes (Figure 2).

To obtain more definitive evidence for the biochemical function of fill, it was overexpressed in E. coli, and the $\mathrm{His}_{6}$-tagged FilI protein was purified to examine its enzymatic function. According to the previously published protocols for Agrobacterium (More et al., 1996), S-adenylmethionine, cell-free extract of $M$. harundinacea 6 Ac and the purified FilI protein were combined for an in vitro AHL synthesis assay. Six major products were detected by ESI-MS analysis with MS peaks at $\mathrm{m} / \mathrm{z}$ 318.2, 284.2, 346.2, 374.2, 274.2 and 302.3 (Figure 3a). The ion intensities were substantially increased compared with the reaction mixtures without Fill (Figure 3b); additionally, the six compounds all generated the characteristic HSL ion $\mathrm{m} / \mathrm{z} 102$ in the tandem ESI-MS (Figure 4, and Supplementary Figures S3 and S4). Therefore FilI was determined to be a methanogenic AHL synthase.

Structure elucidation of the Fill synthetic AHLs To determine the chemical structures of the FilIsynthesized AHLs, these compounds were purified by high-performance liquid chromatography (HPLC) for tandem ESI-MS analysis. The three compounds (1: $\mathrm{m} / \mathrm{z} 374.2 ; 2: \mathrm{m} / \mathrm{z} 346.2$; and $3: \mathrm{m} / \mathrm{z} 318.2$ ) synthesized by FilI all generated a fragment ion of $\mathrm{m} / \mathrm{z} 102$, the AHL characteristic ion product. Meanwhile a concomitant fragment ion of $\mathrm{m} / \mathrm{z} 146$ frequently occurred in the tandem ESI-MS (Figure 4, and Supplementary Figures S3 and S4), which produced a fragment ion of $\mathrm{m} / \mathrm{z} 102$ in its secondary ESI-MS spectrum, implying a carboxyl group connected to the HSL moiety in the AHLs. Following high-resolution ESI-MS, the molecular formula of the three compounds were determined to be $\mathrm{C}_{19} \mathrm{H}_{33} \mathrm{NO}_{5}$ (Compound-1, m/z 356.24319 [M+H] and $\left.\mathrm{m} / \mathrm{z} \quad 374.25403 \quad\left[\mathrm{M}+\mathrm{H}+\mathrm{H}_{2} \mathrm{O}\right]\right), \quad \mathrm{C}_{17} \mathrm{H}_{29} \mathrm{NO}_{5}$ (Compound-2, $\mathrm{m} / \mathrm{z} \quad 328.21217 \quad[\mathrm{M}+\mathrm{H}]$ and $\left.\mathrm{m} / \mathrm{z} \quad 346.22273 \quad\left[\mathrm{M}+\mathrm{H}+\mathrm{H}_{2} \mathrm{O}\right]\right)$ and $\mathrm{C}_{15} \mathrm{H}_{25} \mathrm{NO}_{5}$ (Compound-3, $\mathrm{m} / \mathrm{z} 300.18159[\mathrm{M}+\mathrm{H}]$ and $\mathrm{m} / \mathrm{z}$ 318.19126 $\left.\left[\mathrm{M}+\mathrm{H}+\mathrm{H}_{2} \mathrm{O}\right]\right)$, respectively. The NMR spectrum of Compound-1 (Supplementary Figure S5) also possessed the characteristic resonance signals of AHL, but not the signal of the carboxyl group. To test the possible carboxyl group in Compound-1 it was treated with a vacuum drying process that mimicked the NMR preparation. Following a low-resolution ESI-MS analysis, the molecular ion $\mathrm{m} / \mathrm{z} 374\left[\mathrm{M}+\mathrm{H}+\mathrm{H}_{2} \mathrm{O}\right]$ was remarkably decreased, whereas the abundance of $\mathrm{m} / \mathrm{z} 312$ $[\mathrm{M}+\mathrm{H}]$ was significantly increased. Gas chromatography mass spectrometry experiment indeed detected $\mathrm{CO}_{2}$ during the process of vacuum drying for Compound-1 $(\mathrm{m} / \mathrm{z} 374)$, thus determining that the undetected carboxylate group by ${ }^{1} \mathrm{H}$ NMR was probably attributed to decarboxylation of Compound-1 during preparation for NMR.

Based on the tandem ESI-MS data, two possible linkages of the carboxyl group, either connected with the $N H$ group or the lactone ring, were predicted in Compound-1. To identify the carboxyl modification site, Compound-1 was further subjected to deuterium substitution by dissolved in $\mathrm{CD}_{3} \mathrm{OD}$ at a volume ratio of $1: 1$ at $-20^{\circ} \mathrm{C}$ for 3 days. This experiment is based on the following assumptions: (1) the exchangeable hydrogen in the carboxyl group can be readily substituted by a deuterium; (2) when the carboxyl group connects with the $\mathrm{NH}$, 4 and 2 exchangeable hydrogens would be present in the HSL-opened and HSL-closed Compound-1 as shown in Supplementary Figure S6a, respectively; (3) when the carboxyl group connects with the lactone ring, an extra exchangeable hydrogen would be present ( 5 in the HSL-opened and 3 in the HSLclosed Compound 1, respectively). Undoubtedly, Supplementary Figure S6b showed 4 and 2 deuterium substitutions, but not 5 and 3 in the $\mathrm{CD}_{3} \mathrm{OD}$ treated, HSL-opened $(\mathrm{m} / \mathrm{z}$ 374) and HSL-closed $(\mathrm{m} / \mathrm{z}$ 356) Compound-1, thus confirming the linkage of the carboxyl group with the amino group. Therefore, Compound-1 (m/z 374) and its two homologues (Compound-2: $\mathrm{m} / \mathrm{z} 346$ and Compound-3: $\mathrm{m} / \mathrm{z} 318$ ) were determined to be $\mathrm{N}$-carboxyl- $\mathrm{C}_{14}-\mathrm{HSL}$, 
a
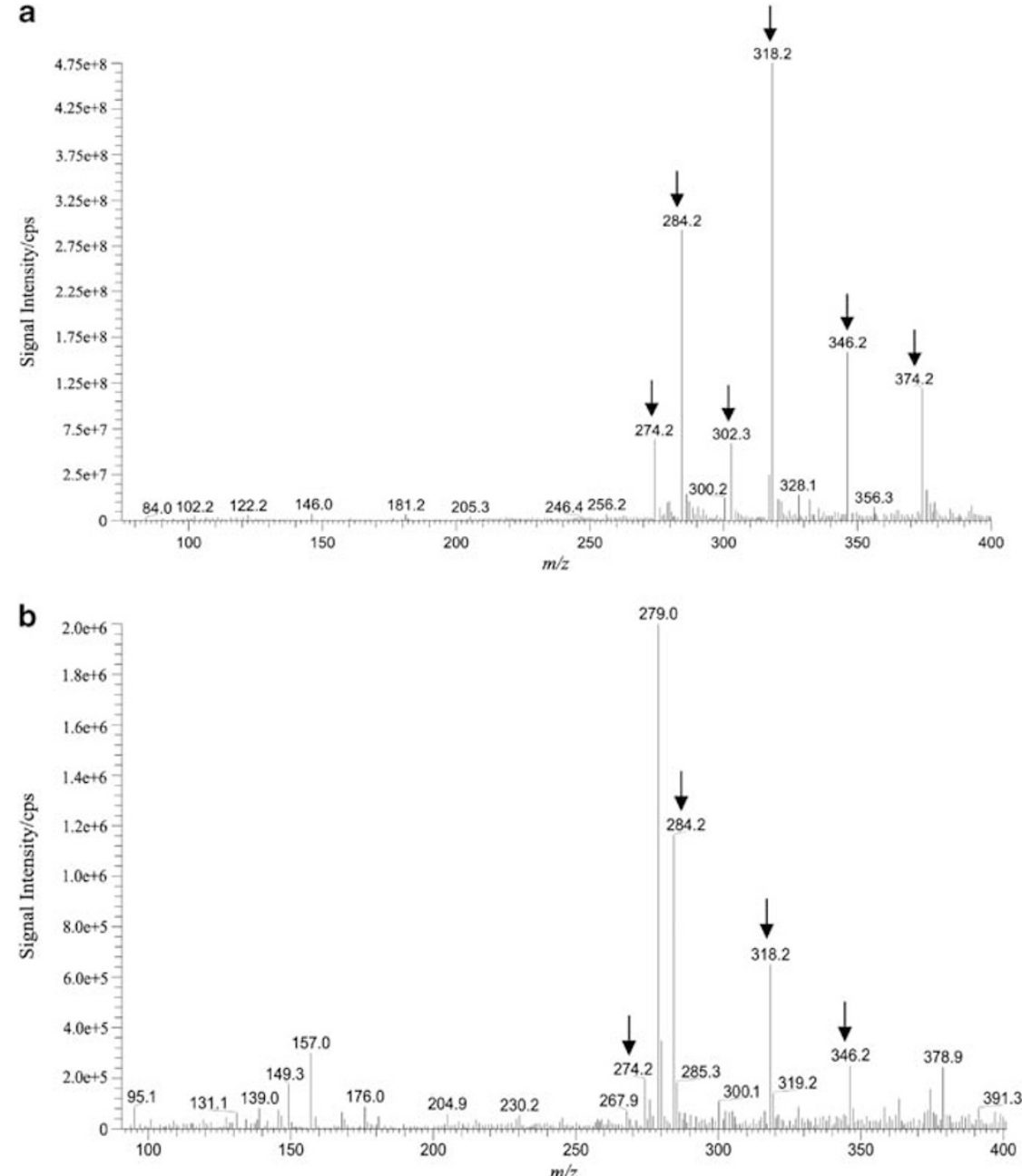

Figure 3 ESI-MS profile of the FilI synthetic mixture. (a) Each of the six compounds indicated by arrows was produced by FilI and formed the characteristic daughter ion $\mathrm{m} / \mathrm{z} 102$ shown in Figure 4, and Supplementary Figures S3 and S4. (b) The cell-free extract of $M$. harundinacea without Fill.

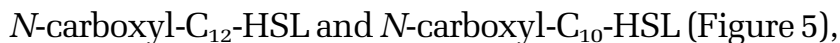
respectively. The three compounds were detected in the spent media as well, indicating that Fill is the synthase for the AHLs produced by M. harundinacea.

Carboxyl-AHLs act as quorum sensing signals in regulating cell form and physiology

To determine whether the FilI-synthesized carboxylAHLs were indeed the signal molecules responsible for the cell density-dependent morphological and physiological changes described above, each of the three purified compounds was added to liquid cultures of $M$. harundinacea at their late log phase or mixed in agar medium at a final concentration of 6-10 nM. With these AHLs, a higher ratio of filamentous cells in the liquid culture after 2-3 weeks and colonies on the agar plates after about 4 weeks were found. By contrast, the cultures supplemented with $\mathrm{m} / \mathrm{z} 256$ and $\mathrm{m} / \mathrm{z}$ 284, the AHLs present in Gram-negative bacteria, isolated in the Fill synthetic mixture, remained almost exclusively in the short cell phenotype and formed very few colonies on plates (Table 1). In addition, a correlation was observed between the filament ratio and the amount of carboxyl-AHLs added (Supplementary Figure S7). These data support the hypothesis that the Fill-synthesized AHLs are likely the signal molecules to mediate cell morphology change of M. harundinacea.

Given the obvious differences in cellular morphology between the short cells and filaments, the two forms were expected to show differences in their metabolism and physiology. During growth on acetate as the sole carbon source as described under Materials and methods, the filaments yielded $31 \%$ more methane, with a concomitant decrease in cellular protein production (38.5\% less), than short cells. Thus the carbon flux in the filamentous cells favoured methane production. Similarly, acetate conversion by filaments possessed a lower apparent $K_{\mathrm{s}}$ value $(0.42 \pm 0.038 \mathrm{mM})$ and higher reaction rate ( $V_{\max }, 0.64 \pm 0.015 \mathrm{mM} \cdot \mathrm{h}^{-1} \cdot \mathrm{mg}^{-1}$ cell protein) compared with the short cells $\left(K_{s}: 0.62 \pm 0.022 \mathrm{mM}\right.$; 

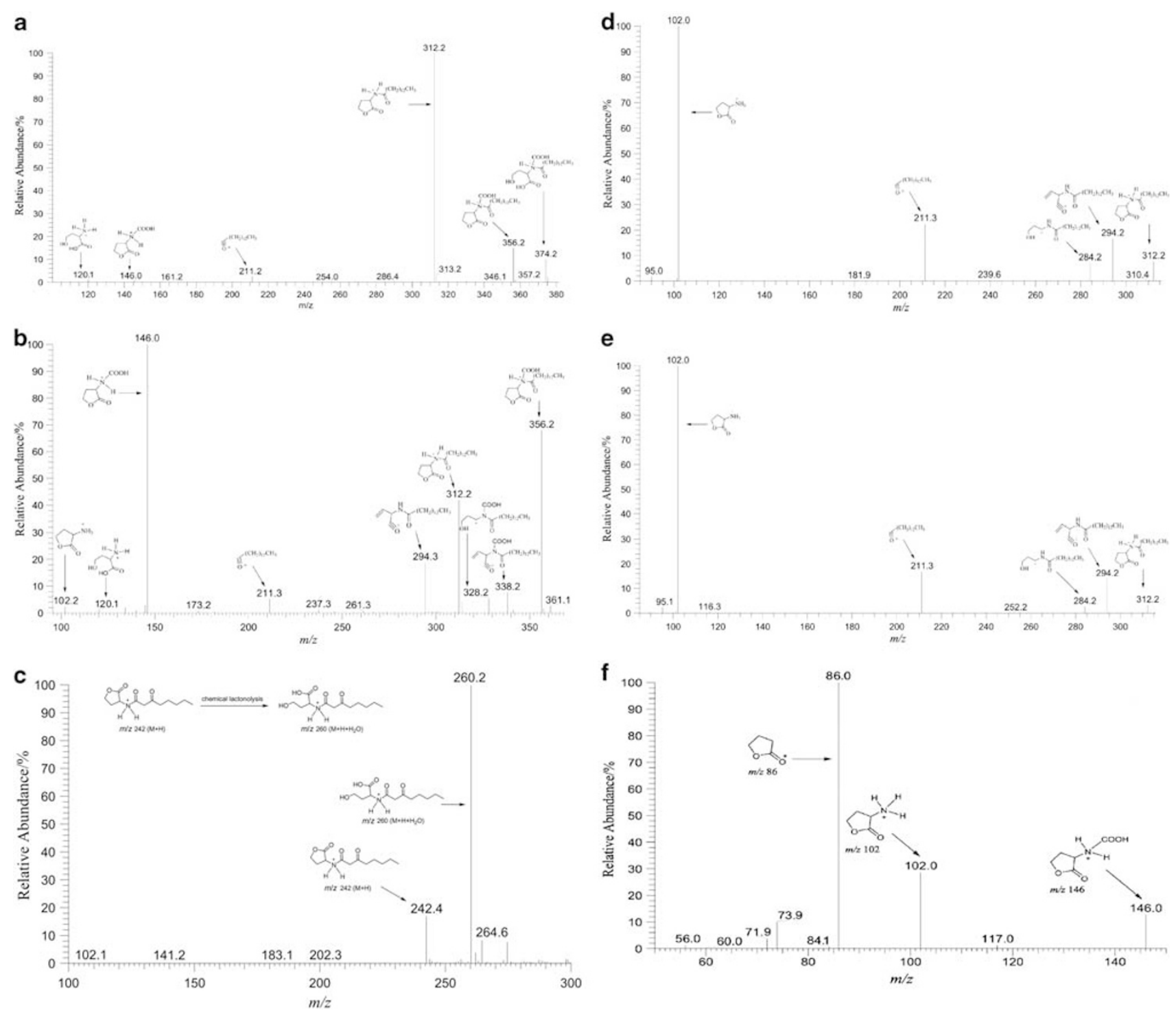

Figure 4 MS/MS daughter scans of Compound-1 (m/z 374) synthesized by Fill and the reference compound. (a) Compound-1 with open HSL ring. (b) Compound-1 with closed HSL ring. (c) $N$ - $\beta$-ketooctanoyl-HSL ( $\mathrm{m} / \mathrm{z} 242$ ) with open HSL ring using ESI-MS analysis.

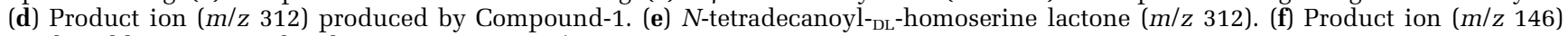
produced by Compound-1 showing an ion at $\mathrm{m} / \mathrm{z} 102$.<smiles>CCCCCCCCCCCCCC(=O)N(C(=O)[O-])C1CCOC1=O</smiles><smiles>CCCCCCCCCCCC(=O)N(C(=O)[O-])C1CCOC1=O</smiles><smiles>CCCCCCCCCC(=O)N(CC(=O)[OH2+])C1CCOC1=O</smiles>

Figure 5 Chemical structures of the Fill synthetic carboxylAHLs. (a) Compound-1: $N$-carboxyl-C $14-\mathrm{HSL}$. (b) Compound-2: $\mathrm{N}$ carboxyl-C ${ }_{12}$-HSL. (c) Compound-3: $N$-carboxyl- $\mathrm{C}_{10}$-HSL. The asterisk refers to the exchangeable hydrogen in each compound.
$V_{\text {max }}: 0.50 \pm 0.028 \mathrm{mM} \cdot \mathrm{h}^{-1} \cdot \mathrm{mg}^{-1}$ ), further indicating the distinct carbon metabolic patterns between the two morphotypes.

To understand how quorum sensing might influence carbon metabolism regulation in $M$. harundinacea, gene expression profiles between the short cells and filaments induced by $N$-carboxyl- $\mathrm{C}_{12}-\mathrm{HSL}$ $(\mathrm{m} / \mathrm{z}$ 346) were compared. The transcriptomes of the two cell types in the late log phase showed that the majority of the genes involved in aceticlastic methanogenesis were downregulated in the filaments (Supplementary Table S1), such as the CO dehydrogenase/acetyl-CoA synthase operon and the electron transfer chain $\mathrm{F}_{420}$ dehydrogenase operon. This result contrasted with the greater conversion of acetate to $\mathrm{CH}_{4}$ in the filaments. However, two acetylCoA synthetase genes (ORF 00570 and 00732), the 
Table 1 Effect of Fill synthetic carboxyl-AHLs on the formation of colonies and filamentous cells of $M$. harundiacea

\begin{tabular}{|c|c|c|c|}
\hline Supplied AHLs & $\begin{array}{c}E S I-M S \\
\text { ions } \\
(\mathrm{m} / \mathrm{z})^{\mathrm{a}}\end{array}$ & $\begin{array}{c}\text { Colonies } \\
\text { per serum } \\
\text { bottle }^{\mathrm{b}}\end{array}$ & $\begin{array}{c}\text { Filamentous } \\
\text { cells } \\
\text { ratio }(\%)^{\mathrm{b}}\end{array}$ \\
\hline None & & $2.5 \pm 1.3$ & 0 \\
\hline$N$-carboxyl-decanoyl-HSL & $318.2 / 300.2$ & $12.8 \pm 5.7$ & $>80$ \\
\hline$N$-carboxyl-lauroyl-HSL & $346.2 / 328.2$ & $5.5 \pm 2.1$ & $>50$ \\
\hline$N$-carboxyl-tetradecanoyl-HSL & $374.2 / 356.2$ & $10.4 \pm 3.8$ & $>90$ \\
\hline$N$-decanoyl-DL-HSL & 256.3 & $2.7 \pm 1.5$ & 0 \\
\hline$N$-lauroyl- ${ }_{\mathrm{DL}}-\mathrm{HSL}$ & 284.2 & $7.1 \pm 3.6$ & 0 \\
\hline
\end{tabular}

Abbreviations: AHL, acyl homoserine lactone; ESI-MS, electrospray ionization mass spectrometry.

a Ion structure with an open/closed lactone ring.

bData from three replicates.

key components for the initial step of the aceticlastic methanogenesis pathway, were both upregulated in the filaments. Genes involved in cellular biosynthetic reactions (encoding pyruvate:ferredoxin oxidoreductase and phosphoenol-pyruvate synthase), transcription (RNA polymerase) and translation (elongation factors) were also downregulated in the filaments, which was consistent with their lower biomass yield. The genes for a number of $\mathrm{F}_{420^{-}}$ dependent enzymes were all highly expressed in the filaments, possibly a cause for the increased autofluorescence. Although, an outer-membrane protein and a filament induction protein were highly expressed in the filaments, these were likely consequences of the specific increase in the production of structural proteins found in the filament shell. As expected, qPCR confirmed the differential expression of each of these genes in the two cell types (Supplementary Table S1).

\section{Discussion}

The AHL-based quorum sensing regulation had previously only been described in Bacteria. In this study, a methanogenic archaeon $M$. harundinacea was shown to use modified AHL molecules, carboxyl-AHLs, to regulate a density-dependent cell behaviour and carbon flux. In order to rule out the possibility that the detected AHLs might have resulted from bacterial contamination, as methanogens frequently live in consortia with syntrophic bacteria for conversion of fatty acids to methane, extensive studies confirmed the purity of the culture. No growth was observed following extensive incubation with rich media in the absence of a substrate for methanogens as well as in the presence of high concentrations of the archaea-specific antibiotics. In addition, PCR amplification of bacterial $16 S$ rRNA genes repeated negative results.

Like many bacterial AHL producers, M. harundinacea possesses a luxI-luxR orthologue, called fill-filR, in the genome. While the putative FilR possesses a homologue to the bacterial LuxR family on the basis of the receiver domains, it groups with the other types of regulators in other methanogen genomes and forms a distinct clade at $23.5 \%$ aminoacid sequence identity with bacterial LuxRs. This suggests a divergent evolution of methanogenic FilR and bacterial LuxRs.

Similar to Gram-negative bacteria, M. harundinacea produces AHLs with a luxI orthologue, fill. Unlike the bacterial AHLs, the three FilI-synthesized-AHLs all possess an extra carboxyl moiety on the $\mathrm{N}$ atom of the HSL ring. Thus, the methanogenic archaeon uses modified bacterial AHLs as the quorum sensing signals.

A similar AHL with a $\mathrm{m} / \mathrm{z} 318$ was also detected in cultures of Methanosarcina mazei and Methanothermobacter thermautotrophicus. In addition, using the fill gene as a probe and a similarity threshold of $0.01 \mathrm{e}$, several signal transduction histidine kinase-encoding genes were identified in the genome sequences of other methanogens showing cell assembly phenotypes (Supplementary Table S2), such as M. mazei (Robinson, 1986), Methanosaeta concilii, Methanosaeta thermophila (Ahring et al., 1991) and Methanospirillum hungatei. These organisms undergo morphological changes or aggregation in a density-dependent manner (Xun et al., 1988). This suggests that AHLs may be the primary quorum sensing molecules used by methanogenic archaea. In addition, bacterial ACP gene homologies are found in some methanogens, such as M. hungatei and Candidatus Methanoregula boonei (Supplementary Table S2), although the concurrence of the ACP with fill gene homologies is not observed in methanogenic genomes. Methanogens may gain bacterial genes that are beneficial for environmental adaptation during evolution. Thus quorum sensing appears to be a universal regulation mechanism in prokaryote.

Finally, Methanosaeta species function as the crucial components of anaerobic digesters, not only for their ability to implement aceticlastic methanogenesis (Jetten et al., 1992), but also for their ability to form filaments that promote the formation of up-flow anaerobic sludge bed granules by serving as a scaffold for attachment by other organisms (Li et al., 2008). Therefore, the methanogenic AHLs might be useful for improving sludge granulation; much like that bacterial AHLs are used to promote phenol degradation in bioreactors (Valle et al., 2004).

\section{Acknowledgements}

We thank R Thauer for valuable discussions on the science impact of the study; Y Che for suggestion on chemical structure determination; and J Merritt for thoroughly editing the manuscript and G Xia for assistance with fluorescence imaging. This work was supported by National Natural Science Foundation of China under numbers 30621005, 30830007 and 31000011, and the Innovation Project of the PUMC Youth Foundation. 


\section{References}

Ahring BK, Alatriste-Mondragon F, Westermann P, Mah RA. (1991). Effects of cations on Methanosarcina thermophila TM-1 growing on moderate concentrations of acetate: production of single cells. Appl Microbiol Biotechnol 35: 686-689.

Cataldi TR, Bianco G, Abate S. (2008). Profiling of $N$-acylhomoserine lactones by liquid chromatography coupled with electrospray ionization and a hybrid quadrupole linear ion-trap and Fourier-transform ioncyclotron-resonance mass spectrometry (LC-ESI-LTQFTICR-MS). J Mass Spectrom 43: 82-96.

Cataldi TR, Bianco G, Abate S. (2009). Accurate mass analysis of $N$-acyl-homoserine-lactones and cognate lactone-opened compounds in bacterial isolates of Pseudomonas aeruginosa PAO1 by LC-ESI-LTQFTICR-MS. J Mass Spectrom 44: 182-192.

Cha C, Gao P, Chen YC, Shaw PD, Farrand SK. (1998). Production of acyl-homoserine lactone quorum-sensing signals by Gram-negative plant-associated bacteria. Mol Plant Microbe Interact 11: 1119-1129.

Doddema HJ, Vogels GD. (1978). Improved identification of methanogenic bacteria by fluorescence microscopy. Appl Environ Microbiol 36: 752-754.

Frommberger M, Hertkorn N, Englmann M, Jakoby S, Hartmann A, Kettrup A et al. (2005). Analysis of $N$ acyl homoserine lactones after alkaline hydrolysis and anion-exchange solid-phase extraction by capillary zone electrophoresis-mass spectrometry. Electrophoresis 26: 1523-1532.

Gobert GN, Moertel LP, Brindley J, McManus DP. (2009). Developmental gene expression profiles of the human pathogen Schistosoma japonicum. BMC Genomics 10: 128-146.

Hastings JW, Greenberg EP. (1999). Quorum sensing: the explanation of a curious phenomenon reveals a common characteristic of bacteria. J Bacteriol 181: 2667-2668.

Hwang I, Li PL, Zhang L, Piper KR, Cook DM, Tate ME et al. (1994). TraI, a LuxI homologue, is responsible for production of conjugation factor, the Ti plasmid $\mathrm{N}$-acyl homoserine lactone autoinducer. Proc Natl Acad Sci USA 91: 4639-4643.

Jetten M, Stams A, Zehnder A. (1992). Methanogenesis from acetate: a comparison of the acetate metabolism in Methanothrix soehngenii and Methanosarcina spp. FEMS Microbiol Let 88: 181-197.

Li J, Hu B, Zheng P, Qaisar M, Mei L. (2008). Filamentous granular sludge bulking in a laboratory scale UASB reactor. Biores Technol 99: 3431-3438.

Ma K, Liu X, Dong X. (2006). Methanosaeta harundinacea, sp. nov., a novel acetate-scavenging methanogen isolated from a UASB reactor. Int J Sys Evol Microbiol 56: $127-131$.

More MI, Finger D, Stryker JL, Fuqua C, Eberhard A, Winans SC. (1996). Enzymatic synthesis of a quorum-sensing autoinducer using defined substrates. Science 272: 1655-1658.

Ortori CA, Atkinson S, Chhabra SR, Cámara M, Williams P, Barrett DA. (2007). Comprehensive profiling of $N$-acylhomoserine lactones produced by Yersinia pseudotuberculosis using liquid chromatography coupled to hybrid quadrupole-linear ion trap mass spectrometry. Anal Bioanal Chem 387: 497-511.

Paggi RA, Martone CB, Fuqua C, De Castro RE. (2003). Detection of quorum sensing signals in the haloalkaliphilic archaeon Natronococcus occultus. FEMS Microbiol Let 221: 49-52.

Pearson JP, Passadori L, Iglewskit BH, Greenberg EP. (1995). A second $N$-acylhomoserine lactone signal produced by Pseudomonas aeruginosa. Proc Natl Acad Sci USA 92: 1490-1494.

Robinson RW. (1986). Life cycles in the methanogenic archaebacterium Methanosarcina mazei. Appl Environ Microbiol 52: 17-27.

Sharif DI, Gallon J, Smith CJ, Dudley E. (2008). Quorum sensing in Cyanobacteria: $N$-octanoyl-homoserine lactone release and response, by the epilithic colonial cyanobacterium Gloeothece PCC6909. ISME J 2: 1171-1182.

Steindler L, Venturi V. (2007). Detection of quorumsensing $N$-acyl homoserine lactone signal molecules by bacterial biosensors. FEMS Microbiol Lett 266: 1-9.

Sun J, Daniel R, Wagner-Döbler I, Zeng AP. (2004). Is autoinducer-2 a universal signal for interspecies communication: a comparative genomic and phylogenetic analysis of the synthesis and signal transduction pathways. BMC Evol Biol 4: 36-46.

Valle A, Bailey MJ, Whiteley AS, Manefield M. (2004). Nacyl- ${ }_{-}$-homoserine lactones (AHLs) affect microbial community composition and function in activated sludge. Environ Microbiol 6: 424-433.

Val DL, Cronan Jr JE. (1998). In vivo evidence that $S$-adenosylmethionine and fatty acid synthesis intermediates are the substrates for the luxI family of autoinducer synthases. J Bacteriol 180: 2644-2651.

Waters CM, Bassler BL. (2005). Quorum sensing: cell-tocell communication in bacteria. Annu Rev Dev Biol 21: 319-346.

Xun LY, Boone DR, Mah RA. (1988). Control of the life cycle of Methanosarcina mazei S-6 by manipulation of growth conditions. Appl Environ Microbiol 54: 2064-2068.

Yu Y, Lee C, Kim J, Hwang S. (2005). Group-specific primer and probe sets to detect methanogenic communities using quantitative real-time polymerase chain reaction. Biotechnol Bioeng 89: 670-679.

Zhou J, Bruns MA, Tiedje JM. (1996). DNA recovery from soils of diverse composition. Appl Environ Microbiol 62: $316-322$.

Supplementary Information accompanies the paper on The ISME Journal website (http://www.nature.com/ismej) 